\title{
Radiological Evaluation of the Therapeutic Response of Malignant Diseases: Status Quo, Innovative Developments and Requirements for Radiology
}

\section{Radiologische Beurteilung des Therapieansprechens maligner Erkrankungen: Status quo, innovative Entwicklungen und Anforderungen an die Radiologie}

Authors

Affiliation

\author{
A. J. Höink, W. Heindel, B. Buerke
}

Department of Clinical Radiology, University Hospital Münster

\author{
Key words \\ - biological effects \\ - PET-CT \\ treatment effects \\ - MR imaging \\ - CT \\ - segmentation
}

received 13.3.2014

accepted $\quad 8.5 .2014$

Bibliography

Dol http://dx.doi.org/

10.1055/s-0034-1366741

Published online: 14.8.2014

Fortschr Röntgenstr 2014; 186 :

927-936 @ Georg Thieme

Verlag KG Stuttgart · New York ISSN 1438-9029

\section{Correspondence \\ Dr. Anna J. Höink}

Institut für Klinische Radiologie, Universitätsklinikum Münster Albert-Schweitzer-Campus 1, Gebäude A1

48149 Münster

Germany

Tel.: ++49/251/8347310

Fax: ++49/2 51/8347312

anna.hoeink@ukmuenster.de

\section{Abstract \\ $\nabla$}

In consequence of the rapid development of newer targeted and personalized tumor therapies, radiology as an essential component of the treatment concept of numerous malignant diseases needs to improve in order to adequately capture and evaluate the effects, but also the side effects of these novel therapeutic agents. The early recognition of therapy response or failure is crucial for the optimal planning of the further treatment and can therefore have direct impact on the chances of recovery and the survival time of oncological patients. In previous years, the goal of medical imaging was to just qualitatively assess the increase or reduction in the size of tumors and their metastases, which was often achieved by a simple subjective estimation of the tumor findings by the diagnosing radiologist. Nowadays, radiology is faced with the challenge of evaluating changes during therapy quantitatively and of visualizing therapeutic effects that are more discrete (e.g. necrosis, altered tumor perfusion). The importance of an adequate assessment of therapy response is further underlined by the fact that in these days, a good portion of oncological patients are enrolled in clinical trials, in which the quantitative radiological evaluation of malignant disorders is an important surrogate parameter. On the basis of this development, the demands for radiology to provide more sophisticated assessments of therapy response and documentation of imaging findings have been constantly growing. The following article provides an overview of the established and still widely spread but in particular also the latest imaging modalities and evaluation criteria with regard to oncological diseases as well as of the increasing demands on radiology that result from these developments. Beyond that, future advancements in tumor imaging are taken into account and the new challenges these developments will bring are discussed.

Key points:

- In the era of personalized medicine, evaluation criteria that are individually adapted to the respective patient are required.

- Radiology needs to substantially contribute to oncological treatment concepts and the evaluation of therapeutic response.

Citation Format:

- Höink AJ, Heindel W, Buerke B. Radiological Evaluation of the Therapeutic Response of Malignant Diseases: Status Quo, Innovative Developments and Requirements for Radiology. Fortschr Röntgenstr 2014; 186: $927-$ 936

\section{Zusammenfassung \\ $\nabla$}

Bedingt durch die rasante Entwicklung immer neuer, zielgerichteter und personalisierter Tumortherapeutika ist auch die Radiologie als wesentlicher Bestandteil im Behandlungskonzept zahlreicher maligner Erkrankungen gefordert, sich ständig weiterzuentwickeln, um die Effekte, aber auch die Nebenwirkungen dieser neuartigen Therapeutika adäquat erfassen und bewerten $\mathrm{zu}$ können. Die frühzeitige Erkennung eines Therapieansprechens oder -versagens ist essenziell für die optimale Planung der weiteren Behandlung und kann damit unmittelbare Auswirkungen auf die Heilungschancen und die Überlebenszeit onkologischer Patienten haben. Bestand die Aufgabe der Bildgebung in früheren Jahren im Wesentlichen darin, ein Größenwachstum oder eine -abnahme von Tumoren und deren Metastasen rein qualitativ zu erfassen, was vielfach durch die einfache subjektive Abschätzung des Tumorbefundes durch den Radiologen erfolgte, so steht sie nun vor der Herausforderung, Änderungen 
unter Therapie quantitativ zu erfassen und auch diskretere Therapieeffekte (wie z. B. Nekrosen oder eine veränderte Tumorperfusion) zu visualisieren. Die adäquate Beurteilung des Therapieansprechens gewinnt zusätzlich durch die Tatsache an Bedeutung, dass ein Großteil der onkologischen Patienten heutzutage in klinische Studien eingeschlossen ist, für die die quantitative radiologische Bewertung maligner Erkrankungen einen wichtigen Surrogatparameter darstellt. Auf Basis dieser Entwicklung sind in den letzten Jahren die Forderungen an die Radiologie hinsichtlich einer immer aufwändigeren Auswertung des Therapieansprechens und der Befunddokumentation ständig gewachsen. Im Folgenden soll ein Überblick sowohl über die etablierten und nach wie vor weit verbreiteten, insbesondere aber über die neuesten bildgebenden Methoden und Bewertungskriterien onkologischer Erkrankungen gegeben werden. Darüber hinaus werden zukünftige Entwicklungen in der bildgebenden Tumordiagnostik in den Fokus genommen und es wird diskutiert, welche neuen Herausforderungen diese Entwicklungen für die Radiologie mit sich bringen.

\section{Introduction}

$\nabla$

Objective radiological assessment of the therapeutic response of malignant diseases is essential for the planning of the further oncological treatment approach: success of a therapy can either be assured early, and effective treatment can be appropriately continued, or in the case of a failure of the therapy, the treatment regime can be changed as soon as possible in order to improve the likelihood of a cure and resulting survival time for the patient, as well as to avoid unnecessary costs of treatment.

Tomography is mostly used in both standard clinical procedures as well as in oncological studies for radiological determination of tumor size and its changes under therapy. Pure dimensioning (through subjective estimation or manual measurement) of malignant lesions has long been established as a means to determine the therapeutic effects of classic cytotoxic chemotherapies. These usually result in irreversible damage in cells with a high rate of mitosis, so that malignant cells are destroyed, leading to a reduction of tumor size which can then be radiologically measured [1 - 3]. Until a few years ago, radiological assessment of malignant lesions relied upon the analysis of analog image data, i.e. $\mathrm{X}$-ray images; therefore, due to a lack of calibration, reliable dimensioning was frequently not possible. Occasionally, evaluation was performed only qualitatively and was thus highly dependent on the subjective impression and experience of the examining radiologist. Absent the possibility of digitizing the image data, measurements could not be documented and stored, and were thus not reproducible. Such problems could be addressed only with the introduction of digital imaging systems (Picture Archiving and Communication System: PACS).

An additional challenge for imaging was the fact that many modern tumor therapies such as angiogenesis inhibitors (e. g. Bevacizumab, Aflibercept) do not result directly in cell death, but rather prevent further tumor growth by preventing or restricting the supply of nutrients to the tumor [4]. In order to determine such cytostatic therapeutic effects (change in tumor perfusion, development of necrosis, etc.), other assessment criteria are required apart from simple size determination, including other imaging modalities. These include, for example, CT- or MR-supported diffusion and perfusion measurements or other functional imaging procedures enabling the evaluation of various stages in the cell cycle of malignant tumors (e.g. imaging of cell proliferation, apoptosis, hypoxia) [5].

\section{Imaging Modalities \\ $\nabla$}

The type of imaging modality can either be defined based on purely physical aspects, that is, according to means of image acquisition (acoustic impedance, magnetic resonance, radiation absorption, etc.), or according to the type of information provided by the respective modality. Imaging processes have developed substantially in recent years: on the one hand, the spatial resolution of radiological processes has continually improved, so that, for example, using high-resolution (HR) CT and MRI it is possible to demonstrate anatomical and morphological information in the sub-millimeter range. At the same time, an increasing number of functional parameters such as tissue perfusion (contrast-enhanced ultrasonography [CEUS], CT and MR perfusion), metabolic processes in the tissue (MR spectroscopy) or brain activation (functional MRT) can be imaged, illustrated and further integrated into routine diagnostic examinations. Recently, methods that can illustrate processes on the molecular level have been increasingly combined in the field of optical imaging (FRI: fluorescence reflectance imaging, FMT: fluorescence-mediated tomography) as well as nuclear medicine techniques (PET: positron emission tomography, SPECT: single photon emission CT). Ideally, these modalities can obtain substantial information simultaneously in hybrid systems [6].

This overview will examine the modalities most commonly used in the clinical routine to assess the therapeutic response of oncological diseases, i. e. CT, MRI and sonography. Projection radiography was consciously excluded from consideration, since this method is notably inferior to CT with respect to detail; likewise, the RECIST guidelines, for example, clearly recommend CT over projection radiography [7].

\section{Measurement Methods \\ $\nabla$}

In principle, the criteria used for evaluating the therapeutic response must meet important prerequisites: they must be quantitative, objectifiable and reproducible in order to reliably substantiate the progression of a disease, thus providing indirect indication of the patient's prognosis [8]. Moreover, in numerous clinical studies, the time in which no tumor progression can be ascertained is an important criterion in assessing the effectiveness of a tested therapy (progression-free survival: PFS) [4]; thus exact quantitative evaluation is extremely important here.

Measurement methods developed in recent years can be classified into three groups:

- The first group includes procedures that only determine tumor size (one-, two- and three-dimensionally).

- The second group is made up of methods especially developed for a specific tumor entity in order to adequately account for its particular characteristics (e.g. arterial con- 
trast medium uptake, necrosis); this category comprises one- and two-dimensional sizing as well as functional criteria.

- Functional measurement parameters demonstrated using different imaging modalities (MRI, PET, etc.) comprise the third group.

All measuring procedures are founded on changes malignant tumors or metastases undergo as a result of therapy. Prior to systemic or local therapy, a baseline is defined to be compared against additional follow-up measurements. Threshold values have been established for the various criteria; using these, disease process is categorized as completely (complete response) or partially regredient (partial response), stable (stable disease) or progredient (progressive disease).

Unless explicitly stated otherwise, the criteria below relate to measurements obtained from CT data sets in the transverse plane.

\section{Determining Tumor Size \\ $\nabla$}

\section{WHO criteria}

In 1979, the World Health Organisation (WHO) developed the first standardized measuring methods to determine the therapeutic response of solid tumors using radiological procedures [9]. The size of a lesion is determined by the product of its greatest longitudinal diameter times the largest perpendicular diameter, resulting in a two-dimensional measuring procedure. If several lesions are present, then their products are added up. These criteria have disadvantages: the type of imaging modality is not specified, there is no minimum definition of the lesions, and there is no specification of the minimum and maximum number of lesions to be determined [10] ( $\bullet$ Fig. 1).

Since the threshold value for a disease progression with an increase of $25 \%$ of the sum of all malignant lesions is relatively low, compared to other evaluation criteria (see below), WHO criteria tend to prematurely evaluate an increase in lesion size as a progression in disease. Nevertheless it could be demonstrated that a positive therapeutic response based on WHO criteria could be correlated with an improved total survival time of the affected patient [11].

\section{RECIST}

The Response Evaluation Criteria in Solid Tumors (RECIST) were first published in 2000 (RECIST 1.0) [12] and thoroughly revised in 2009 (RECIST 1.1) [7]. Unlike WHO criteria, RECIST rules are based on one-dimensional measurements.

According to RECIST 1.1, a pre-therapeutic baseline examination should record a maximum of five malignancies, called target lesions (max. two lesions per organ), which are tracked and measured during subsequent follow-up controls. The greatest longitudinal diameter of the lesions should be measured; in the case of suspected lymph nodes, the largest transverse diameter is measured. To qualify as a target lesion, the diameter must be at least $10 \mathrm{~mm}$, in the case of lymph nodes, the diameter should be at least $15 \mathrm{~mm}$. Any additional non-measurable lesions $(<10 /<15 \mathrm{~mm}$, pleural effusions, etc.) are recorded as non-target lesions and assessed purely qualitatively ( $\bullet$ Fig. 2 ).

RECIST 1.1 indicates CT as the most appropriate examination method for evaluation; however, MRI is mentioned as an alternative and ${ }^{18} \mathrm{~F}$-Fluordeoxyglucose PET $\left({ }^{18} \mathrm{~F}-\mathrm{FDG}\right.$ PET) is included as well. The maximum slice thickness is also specified in order to further standardize assessments. The disadvantage of this method is the absence of detection of necrosis [13] ( $\bullet$ Table 1 ).

\section{Volumetric analysis}

Computer-based volume measurement of malignant lesions offers an alternative to the one- and two-dimensional measurement procedures described above. Although in the previous decade, volumetric segmentation was initially technically feasible only with respect to pulmonary nodules, current algorithms likewise permit volume measurement of lymph nodes and tumors, although these are more difficult to define due to the lower density differences with the surrounding tissue [14]. According to some studies, semi-automated volumetric analysis correlates better with the quantity of tumor cells $[15,16]$, and reduces the negative influence of manually performed one- and two-dimensional measurements, thus permitting high reproducibility while lowering inter- and intra-observer variability [17]. Further, it could be shown that the therapeutic response of malignant tumors can be better estimated using volumetric analysis than with manual linear methods [18] ( $\bullet$ Fig. 3).
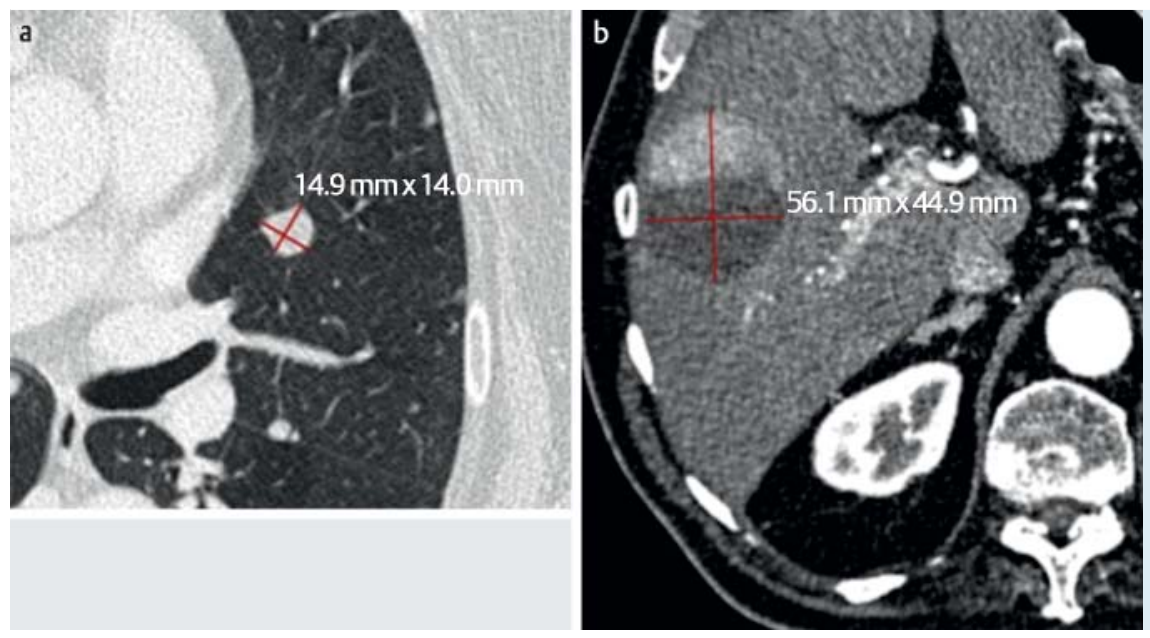

Fig. 1 Identification of two malignant lesions according to WHO guidelines. a 61-year-old patient with pulmonary metastases of a synovial sarcoma. Determination of the longest diameter $(14.9 \mathrm{~mm})$ and its longest perpendicular diameter $(14.0 \mathrm{~mm})$ of a metastasis in the superior lobe of the left lung. According to the WHO guidelines, this results in an area of $208.6 \mathrm{~mm}^{2}$. b 82 -year-old patient suffering from hepatocellular carcinoma (HCC) that is treated by transarterial chemoembolization (TACE). According to the WHO guidelines, the whole lesion, i. e. both its vital and its necrotic part, is measured, which results in an area of $2,518.9 \mathrm{~mm}^{2}$ $(56.1 \times 44.9 \mathrm{~mm})$. 

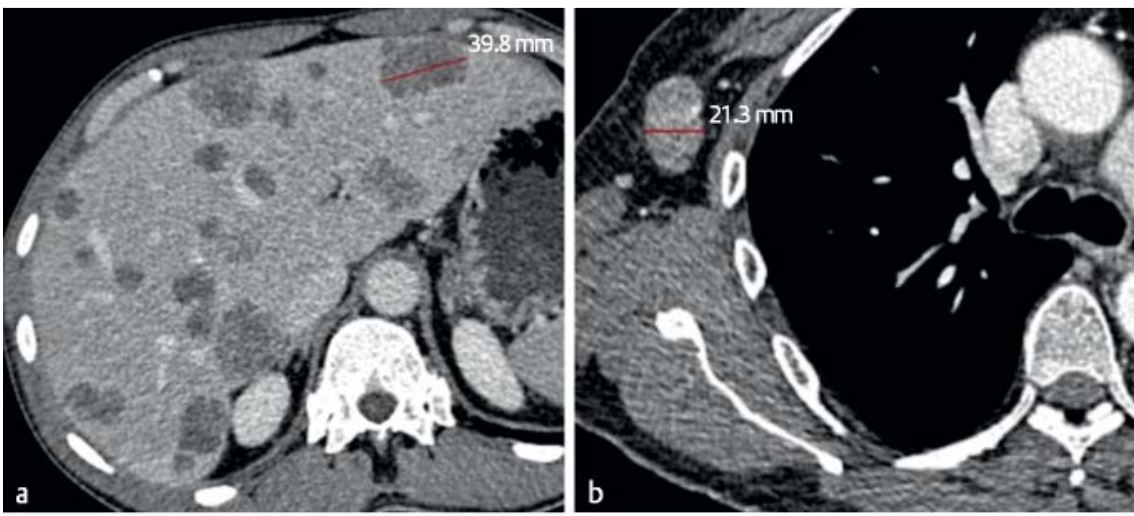

Fig. 2 Identification of two malignant lesions according to RECIST. a 52-year-old patient with a hematogenously and lymphogenously metastasized renal cell carcinoma. The long axis of a hepatic metastasis measured according to the RECIST guidelines is $39.8 \mathrm{~mm}$. b 68 -year-old patient suffering from a lymphogenously and subcutaneously metastasized amelanotic malignant melanoma. The short axis of a lymph node metastasis located in the right axilla is $21.3 \mathrm{~mm}$.
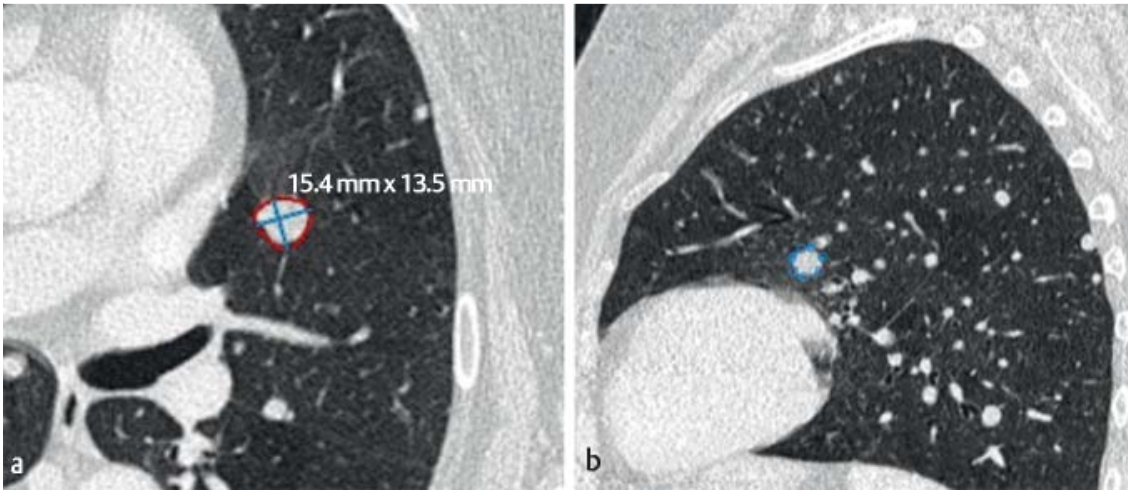

Fig. 3 Semi-automated volumetric analysis of a pulmonary nodule. a Pulmonary metastasis of a synovial sarcoma. In the axially orientated CT image, the external contours of the nodule are defined manually by setting individual points that confine the nodule. A software (mint Lesion ${ }^{\mathrm{TM}}$, Mint Medical $\mathrm{GmbH}$, Heidelberg) interpolates the line between the points. $\mathbf{b}$ Afterwards, the same procedure is repeated in a sagittally (or coronally) orientated CT image in order to define the contours in the third spatial dimension. The volume of this pulmonary nodule, which is calculated by the software, is $1.1 \mathrm{~cm}^{3}$.

Table 1 Criteria for determining tumor size.

\begin{tabular}{|c|c|c|}
\hline characteristics & WHO & RECIST 1.1 \\
\hline publication year & 1979 & 2009 \\
\hline measurement method & two-dimensional & one-dimensional \\
\hline \multirow[t]{2}{*}{ measurable lesions (CT) } & \multirow{2}{*}{$\begin{array}{l}\text { all one- or two-dimensionally meas- } \\
\text { urable lesions }\end{array}$} & lymph nodes \\
\hline & & longitudinal diameter $\geq 10 \mathrm{~mm}$ \\
\hline \multirow[t]{3}{*}{ non-measurable lesions (CT) } & \multirow{3}{*}{$\begin{array}{l}\text { lymphangitic pulmonary metastases, } \\
\text { skin involvement in breast cancer, } \\
\text { etc. }\end{array}$} & lymph nodes \\
\hline & & transverse diameter $\geq 10 \mathrm{~mm}$ and $<15 \mathrm{~mm}$ \\
\hline & & $\begin{array}{l}\text { leptomeningeal dissemination, ascites, pleural/pericardial effusion, } \\
\text { osteoplastic metastases, etc. }\end{array}$ \\
\hline number of lesions & not defined & max. 5 target lesions and max. 2 per organ \\
\hline \multicolumn{3}{|l|}{ therapeutic response } \\
\hline complete remission (CR) & disappearance of all lesions & disappearance of all target lesions, all lymph nodes $<10 \mathrm{~mm}$ \\
\hline partial remission (PR) & decrease in sum of all lesions $\geq 50 \%$ & decrease in sum of diameters of all target lesions $\geq 30 \%$ \\
\hline stable disease (SD) & $\begin{array}{l}\text { decrease in sum of all lesions }<50 \% \\
\text { and increase of sum }<25 \%\end{array}$ & neither CR nor PR nor PD \\
\hline progressive disease (PD) & increase in sum of all lesions $\geq 25 \%$ & $\begin{array}{l}\text { decrease in sum of diameters of target lesions } \geq 20 \% \text { and absolute increase } \\
\text { of sum } \geq 5 \mathrm{~mm} \text {, new lesions }\end{array}$ \\
\hline
\end{tabular}

therapies, such as transcatheter arterial chemoemboliza-

\section{Disease-specific Criteria}

$\nabla$

\section{Hepatocellular carcinoma (HCC): EASL, mRECIST and RECICL}

The above criteria do not take into account the previously mentioned phenomenon that tumors sometimes do not respond to chemotherapy or other therapies by changing in size, but rather only through necrosis, for example. Necrosis as the sole indicator of a therapeutic response is observed in the case of HCC, among others; this carcinoma frequently responds only with a change in tumor vascularization to targeted systemic (e.g. sorafenib) or minimally invasive tion (TACE) or selective internal radiation therapy (SIRT) $[19,20]$.

In order to meet this problem adequately, the European Association for the Study of the Liver (EASL) developed criteria published in 2001, based on two-dimensional measurements of vital tumor components, i.e. the areas that accumulate contrast media in the arterial phase [21].

In 2010, the RECIST guidelines were likewise adapted to include and quantify tumor necrosis; these criteria combine the EASL criteria with those of RECIST and are known as modified RECIST (mRECIST) [22]. According to mRECIST, 

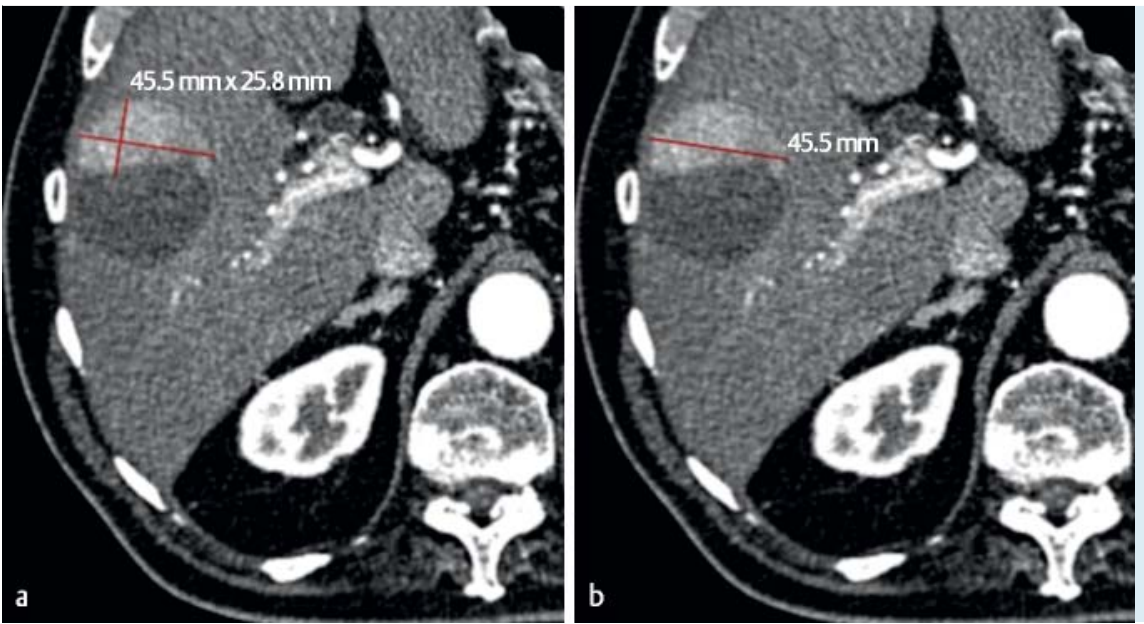

Fig. 4 Measurement of an HCC lesion according to EASL criteria and mRECIST. a Hepatocellular carcinoma (HCC) that is treated by TACE. According to the EASL guidelines, the vital, i. e. the contrast-enhancing part of the tumor is measured. For this purpose, the longest diameter $(45.5 \mathrm{~mm})$ and its longest perpendicular diameter $(25.8 \mathrm{~mm})$ are determined (as in the WHO guidelines). $\mathbf{b}$ mRECIST requires the determination of the long axis of the vital part of the tumor only, which in this case is $45.5 \mathrm{~mm}$.

Table 2 Characteristics of disease-specific criteria.

\begin{tabular}{|c|c|c|c|c|c|}
\hline characteristics & EASL & MRECIST & RECICL & IWC (Cheson) & Choi \\
\hline publication year & 2001 & 2010 & 2009 & 2007 & 2004 \\
\hline tumor entity & \multicolumn{3}{|c|}{ hepatocellular carcinoma (HCC) } & non-Hodgkin's lymphoma (NHL) & gastrointestinal stroma tumor (GIST) \\
\hline $\begin{array}{l}\text { arterial contrast medium uptake/ } \\
\text { necrosis }\end{array}$ & yes & yes & yes & no & (no) \\
\hline density (Hounsfield units) & (no) & (no) & (no) & no & yes \\
\hline tumor marker (laboratory) & no & no & yes & yes & no \\
\hline histology/immunohistochemistry & no & no & (yes) & yes & no \\
\hline FDG PET & no & no & no & yes & no \\
\hline clinical findings & no & no & no & yes & no \\
\hline
\end{tabular}

the longest diameter of the tumor component receiving arterial contrast medium is determined ( $\bullet$ Fig.4).

The Response Evaluation Criteria in Cancer of the Liver (RECICL) which likewise pertain to quantification of vital tumor components were revised in 2009 [23] in order to better measure the response of HCC to minimally ablative and targeted therapies. Tumor markers verified by laboratory results (e.g. $\alpha 1$-fetoprotein [AFP]) are also included in the assessment of the therapeutic response.

\section{Lymphomas: IWC (IWG, IHP, Cheson criteria)}

The initial version of the International Workshop Criteria (IWC) developed by an international working group (IWG) for standardizing the evaluation of the therapeutic response of non-Hodgkin lymphomas (NHL), is based on laboratorydeveloped markers, clinical findings, and radiological assessment of lymphoma manifestations using CT [24]. Beyond that, the revised edition of the IWC, the product of the International Harmonization Project (IHP) also includes Hodgkin's lymphoma and utilizes findings using FDG PET as well as immunohistochemical results, thus enhancing the sensitivity of assessment [25].

The radiological examination of a lymphoma is based on the determination of the sum of the products of the largest diameter and related perpendicular diameter of up to six nodal target lesions. Malignancies with a longitudinal diameter of $>15 \mathrm{~mm}$ or a transverse diameter of $>10 \mathrm{~mm}$ are considered target or measurable lesions. A diameter of $\geq 10 \mathrm{~mm}$ is required for extranodal lesions.
Gastrointestinal stroma tumor (GIST): Choi criteria

Choi criteria were developed to assess the response of GIST to therapy using tyrosine-kinase inhibitors (TKI), since tumors under this therapy frequently exhibit no size reduction, even though they respond well to TKI. Consequently, in addition to tumor size, the criteria take into account the tumor's density in CT (venous phase) [26] ( $\bullet$ Table 2 ).

\section{Limitations}

$\nabla$

The development of the above criteria was an attempt to account for individual problems posed by the related tumor entities. Thus specific tumor characteristics were incorporated into the assessment criteria, such as arterial contrast medium absorption or lack of change in size despite a good therapeutic response; specific biochemical tumor markers were likewise included.

Hitherto there was insufficient substantiated data that could be used to define threshold values for the classification of a therapeutic outcome (response, stable disease, etc.) for the respective criteria. Therefore the limit values are frequently adopted from the RECIST guidelines or only somewhat modified.

The advantages of criteria that particularly address specific tumor entities likewise pose the disadvantage that they cannot be applied to other malignancies. 


\section{Functional Imaging}

$\nabla$

\section{Dynamic contrast-enhanced (DCE) CT / MRI}

\section{and contrast-enhanced ultrasonography (CEUS)}

Angiogenesis is a decisive factor in tumor biology, since it enables malignant tumors to grow and metastasize [27, 28 ]. To date, the reference standard to determine the vascularization level of a tumor is the histopathological measurement of vessel density; however, this poses the disadvantage of invasiveness. Therefore it is of great interest to use imaging methods in conjunction with anti-angiogenic or anti-vascular therapy to determine the vessel density of a tumor and the change in vascularization in order to detect the therapeutic response early and non-invasively.

Dynamic contrast-enhanced (DCE) MRI is the imaging method most commonly used in preclinical and clinical studies to evaluate the effectiveness of vascular disrupting agents (VDA) [29]. Using kinetic parameters such as transfer constant $\mathrm{K}^{\text {trans }}$ and IAUGC (initial area under the gadolinium curve), it is possible to detect the anti-vascular effect of these therapies non-invasively with MRI [30], ( $\bullet$ Fig.5).

Likewise, contrast-enhanced ultrasonography (CEUS) permits real-time visualization of the change of tumor perfusion under therapy with vascular targeting agents (VTA), such as tTF-NGR [31].

Compared to MRI, dynamic CT provides a substantial advantage in that there is a linear relationship between the contrast agent absorption of the tumor and the iodine concentration, thus allowing an absolute quantification of the perfusion [32]. Recent investigations have shown that CT perfusion can therefore be used for quantitative analysis of hemodynamic changes of tumors under therapy [33, 34]. Limitations of CT perfusion are the relatively high radiation dose and limited examination volume, which due to technical and radiationhygienic reasons cannot be as high as desired.

\section{Diffusion-weighted MRI (DWI)}

Diffusion-weighted imaging permits detection of the movement of water molecules in the tissue (Brownian motion). Proton movement in all three spatial dimensions is measured and quantified by the calculation of the apparent diffusion coefficient (ADC), which provides a criterion for the diffusion characteristics of a tissue. Although DWI was initially reserved for the neuroradiological ischemia diagnostics, the development of more rapid echo planar sequences (echo planar imaging: EPI), as well as improved gradient systems and coils have achieved diagnostic image quality in the area of the trunk [35].

Using DWI it is sometimes possible to detect therapy-related changes in the texture of a tumor even before a change in size is observable [5]. Therapy-induced cell death leads to a reduction of cell density, and thus results in an improved diffusion and an increase of the $\operatorname{ADC}[4,36]$. Further, it is possible, particularly in strongly vascularized lesions, to detect perfusion effects using DWI; in order to distinguish these effects from molecular diffusion, it is necessary to acquire images with lower b-values $\left(0-100 \mathrm{~s} / \mathrm{mm}^{2}\right)$, since they are sensitive to perfusion effects [36].

The suitability of DWI as an appropriate biomarker for the assessment of the therapeutic response of malignant diseases has already been confirmed at an open consensus conference during a meeting of the International Society for Magnetic Resonance in Medicine (ISMRM), which took place in 2008 and was sponsored by the National Cancer Institute $(\mathrm{NCI})$. In this context it was stated that there were previously no standardized studies that sufficiently proved the diagnostic potential of DWI and its correlation with histopathological results [37].

\section{MR spectroscopy (MRS)}

${ }^{1} \mathrm{H}-\mathrm{MR}$ spectroscopy enables the detection and quantification of various metabolites of cell metabolism such as choline, N-acetylaspartate (NAA) or citrate in a selected volume element [38]. The specific resonance frequencies for the different metabolites given in parts per million (ppm) are shown on the $\mathrm{x}$-axis of a coordinate system, and the related signal intensities are indicated on the y-axis ( $\bullet$ Fig.6).

Choline metabolism is of particular interest for oncological imaging since elevated levels of phosphocholine (PCho) and total choline-containing compounds (tCho) could so far be detected in almost every tumor entity, which means that these metabolites could be used as a non-invasive biomarker to assess therapeutic response [39].

\section{MR relaxometry}

Steady-state MR imaging is based on the use of so-called blood-pool contrast agents, characterized by a long intravasal hold time. This group of contrast media includes USPIO
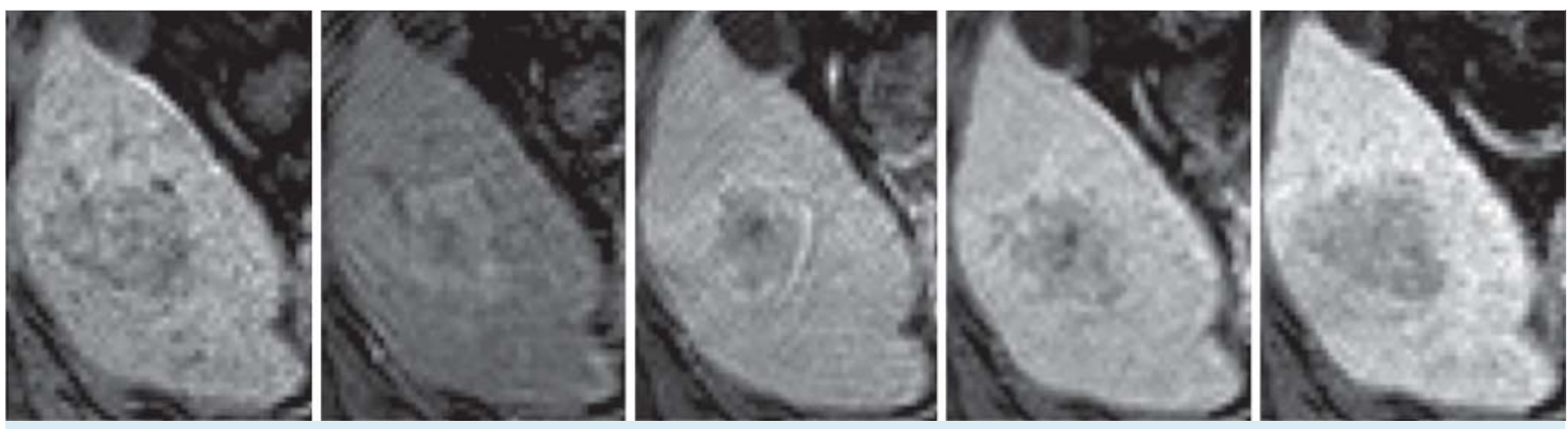

Fig. 5 Dynamic contract-enhanced MRI (DCE MRI) of HCC in the right liver lobe. 65-year-old patient suffering from hepatocellular carcinoma (HCC) in the right lobe of the liver as a result of hepatic cirrhosis due to chronic hepatitis C. The figure displays the dynamic MRI scans (ultrafast T1-weighted gradient echo sequence [THRIVE]) by using a gadolinium-containing i. v. contrast agent (Gadovist ${ }^{\circledR}$ ). The images prior to the application of contrast agent (native) and after 20,60 and 120 seconds as well as after 5 minutes are pictured (from left to right). 
(ultrasmall superparamagnetic iron oxide) particles. The relative blood volume of a tumor can be monitored by quantitatively determining the changes in the transversal relaxation rate $\left(\triangle \mathrm{R} 2^{*}\right)$ induced by the USPIO particles. Determination of the blood volume is based on the assumption that $\triangle \mathrm{R} 2 *$ is proportional to the local blood volume within a defined volume.

Using MR relaxometry it is therefore possible to characterize the vasculature of a tumor non-invasively while also monitoring the effect of anti-angiogenic therapies [40].

\section{Positron emission tomography (PET): EORTC, PERCIST}

The most widely used PET tracer in oncological imaging is ${ }^{18} \mathrm{~F}-\mathrm{FDG}$ [41]; it is metabolized by many malignant tumors and can therefore be used for tumor screening and staging. Beyond, using PET, numerous other components of the cell cycle of malignant tumors can be visualized, such as proliferation, apoptosis or hypoxia (PET using ${ }^{18} \mathrm{~F}$-Thymidine, ${ }^{18} \mathrm{~F}$-Annexine or ${ }^{18} \mathrm{~F}$-Fluoromisonidazole) [5] (๑ Fig. 7). In 1999 the European Organisation for Research and Treatment of Cancer (EORTC) developed guidelines for the evaluation of the therapeutic response of solid tumors using FDG

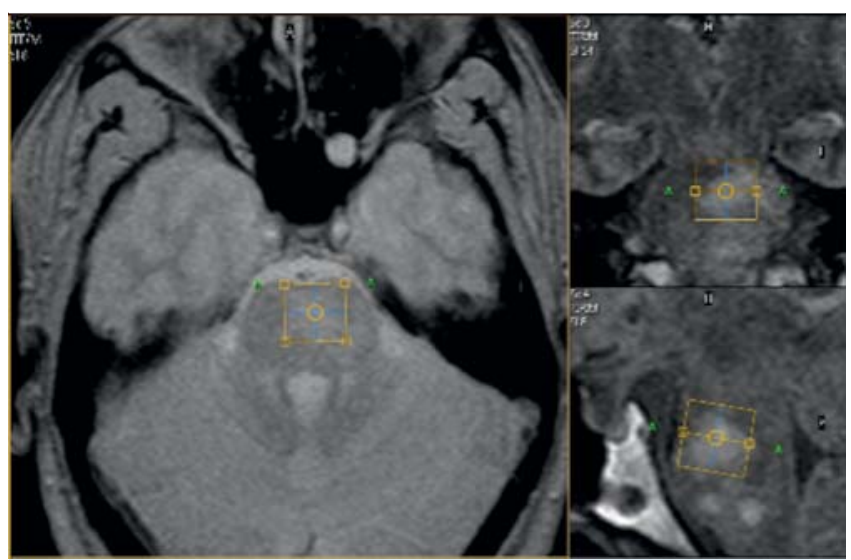

Fig. 6 MR spectroscopy of a suspected lesion in the brainstem. 36-yearold patient with a suspected low-grade malignant glioma in the pons. The MR spectroscopy of this lesion in the brainstem shows elevated values of choline, creatine and myo-inosit but normal lactate and $\mathrm{N}$-acetylaspartate (NAA) values.
PET [42]. The therapeutic result is assessed using the change of the SUV (standardized uptake value) of malignant lesions under therapy.

Additional criteria used to assess the metabolic response of malignant tumors are the PET Response Criteria in Solid Tumors (PERCIST 1.0). The advantages of PERCIST compared to the EORTC guidelines include the combination of morphological and metabolic parameters, i.e. PET is performed as a PET CT, and that the patient's body weight is taken into account to determine glucose metabolism (SUL: lean body mass-normalized SUV). Analogous to RECIST, according to PERCIST, up to five lesions can be measured (max. two per organ) [43].

\section{Measuring Technique \\ $\nabla$}

In clinical studies, radiological assessment of malignancies mostly relies on purely manual evaluation of CT data according to RECIST 1.1 or WHO guidelines. However, if oncological patients are not included in a study, occasionally there is no standardized evaluation of the malignant lesions at all.

Both the steadily growing number of interdisciplinary tumor centers (Comprehensive Cancer Center: CCC), as well as the increasing mobility of cancer patients who are occasionally treated in two or more different centers lead to the radiological assessment of the same patient by different radiologists at various locations. Some single- and multi-center examinations have already demonstrated that this course of action, i. e. manual and/or one-dimensional evaluation of malignant lesions by several radiologists results in high inter- and intra-observer variability $[17,44]$, and that semi-automated determination of multi-dimensional parameters can reduce the rate of misclassification of the therapeutic response [45].

Together with recent advances in CT technology, this knowledge has led to the development of software that can be used to measure and segment lesions semi-automatically (॰ Fig. 8).

In addition to semi-automated measurement of the RECIST diameter or WHO area, various software applications (mint Lesion $^{\mathrm{TM}}$ [Mint Medical GmbH, Heidelberg], syngo ${ }^{\mathrm{TM}}$ RT Oncologist [Siemens Healthcare, Erlangen] among others), also
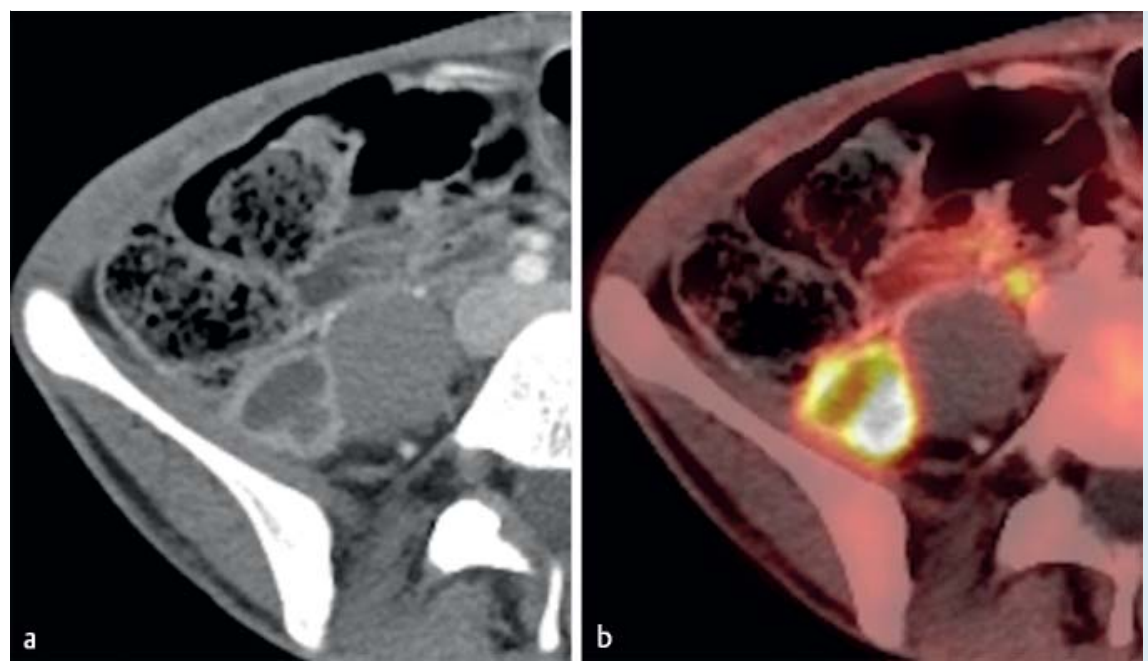

Fig. 7 PET CT of a metabolically active metastasis of a Ewing sarcoma. a 33-year-old patient with a metastasized Ewing sarcoma. Contrast-enhanced CT scan of a partly necrotic, most probably malignant mass adjacent to the right Musculus iliopsoas. b Fusion image (PET $+\mathrm{CT}$ ) of the same lesion that shows a pathologically increased glucose metabolism (SUV max. 11.3), so that the suspected diagnosis of a metastasis can be corroborated. The smaller, metabolically active lesion lying ventral and medial to the right Musculus psoas corresponds to the right ureter. 
offer the option of volumetric evaluation and documentation of malignant alterations.

\section{Conclusion}

$\nabla$

The generic term malignant disease includes a great number of heterogeneous disease entities that differ with respect to their histopathological characteristics (squamous cell / adeno carcinoma, lymphoma, etc.), their localization as well as growth and metastatic behavior. Research in recent years has shown - using up-to-date imaging processes - that relevant differences can be found even within a single tumor entity. These are mostly based on genetic variants, which lead to mutations in proto-oncogenes (KRas, tyrosine-kinases, etc.) or hormone receptors, so that within one tumor type, several genetic subpopulations could arise which to some extent significantly differ from one another [46].
The logical consequence of these insights is that therapy regimes of malignant diseases must constantly adapt to the above-named conditions and therefore new tumor therapies must be continuously developed. New therapeutics often no longer rely on a cytotoxic effect, but rather focus on the molecular characteristics of tumors, by targeting the receptor level, for example.

The developments identified here make it clear that although patients may suffer from the same malignant disease, they sometimes cannot be treated with the identical therapeutic regime; instead the treatment strategy must be adapted to both the special characteristics of the tumor as well as the interindividual differences of the patients. Thus the therapy for a postmenopausal woman suffering from a breast cancer that expresses neither estrogen nor progesterone receptors and is negative for HER2/neu can be significantly different from that for a premenopausal patient with breast cancer that is positive for both hormone receptors and HER2/neu.

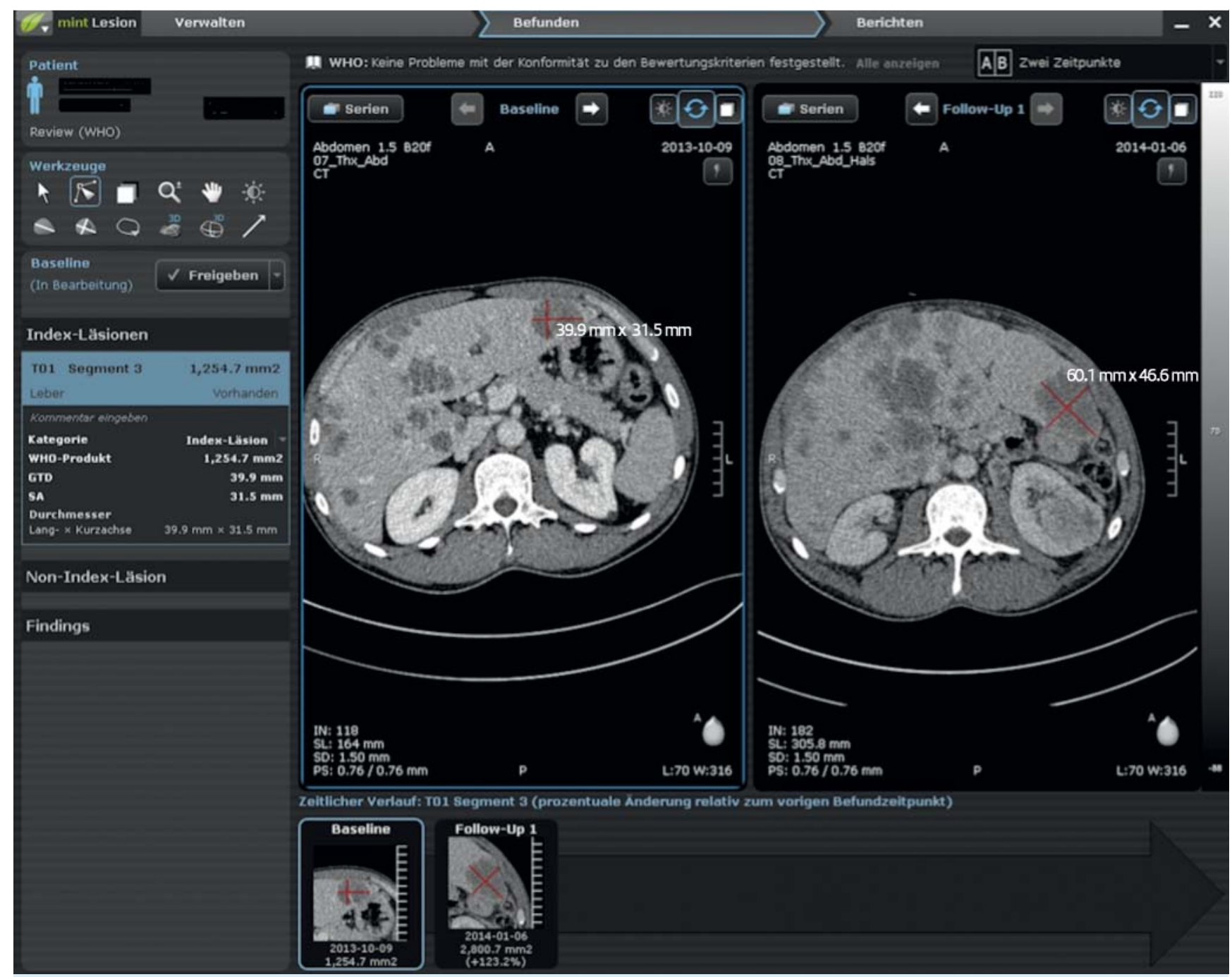

Fig. 8 Semi-automated determination of lesion size according to WHO criteria using mint Lesion ${ }^{\mathrm{TM}}$ (Mint Medical $\mathrm{GmbH}$, Heidelberg). Semi-automatic determination of the size of a hepatic metastasis of a renal cell carcinoma according to the WHO guidelines with the aid of mint Lesion ${ }^{\mathrm{TM}}$ (Mint Medical $\mathrm{GmbH}$, Heidelberg). On the far left, the different measuring tools are displayed beneath the patient data (blackened), which can be selected by means of a mouse click. The different information concerning the selected index lesion (WHO product, long axis etc.) are displayed below. The two $\mathrm{CT}$ images in the middle and on the right show the lesion at baseline and at the time of the first follow-up. Below, the lesion is displayed over the course of time on a timeline and its resizing is indicated in \%. 
As an interdisciplinary interface, radiology is a central component in the concept of oncological treatment of the majority of malignant diseases. Thus there is a constant demand for radiology not only to adapt to new findings in the field of tumor origin and therapy, but also to contribute actively to a better understanding of the genesis of malignant diseases and to the improvement of existing therapy regimes.

It has already been postulated that certain tumor characteristics, which can be demonstrated on a macroscopic and/or functional level using imaging methods, mirror processes of tumor biology on the molecular and cellular level [46]. On the one hand, this fact provides an opportunity to better understand the individual characteristics of tumor biology, while on the other hand, making it clear that the type of therapeutic response detectable using imaging can vary, since it is dependent on these very characteristics ( $\bullet$ Fig. 9). It is clear that evaluation criteria based solely on the assessment of morphological parameters (WHO, RECIST) demonstrate clear limitations in the evaluation of malignancies treated with targeted therapies, since a size increase of a slow-growing tumor indicates disease progression, for example, whereas in a rapidly growing malignoma, it can mean a good therapeutic response [15, 47]. As already explained, those criteria that include functional parameters in assessment represent a significant improvement in ap- proach (EASL, PERCIST, etc.). However, even these criteria in themselves are occasionally not sufficient to adequately assess a therapeutic response.

In the course of the so-called personalized medicine which is constantly evolving based on the innovations mentioned in this overview, nowadays newer criteria are needed that focus individually on the respective tumor entity with its histopathological and molecular features, the nature of the therapeutic agent, the characteristics of the affected patient and the imaging modality used to detect the therapeutic response; all of these attributes must be accounted for in equal measure. Since such criteria can contribute to a closer meshing of (imaging) diagnostics and (oncological) therapy in terms of the principle of theranostics, in addition to radiological biomarkers, special clinical findings and biochemical markers should also be taken into account in order to have a more comprehensive view of the disease response under therapy.

As a result of innovative imaging approaches, radiology therefore needs to establish corresponding imaging-based parameters in order to help shape the oncological therapeutic concept as well as the assessment of the response to therapy.

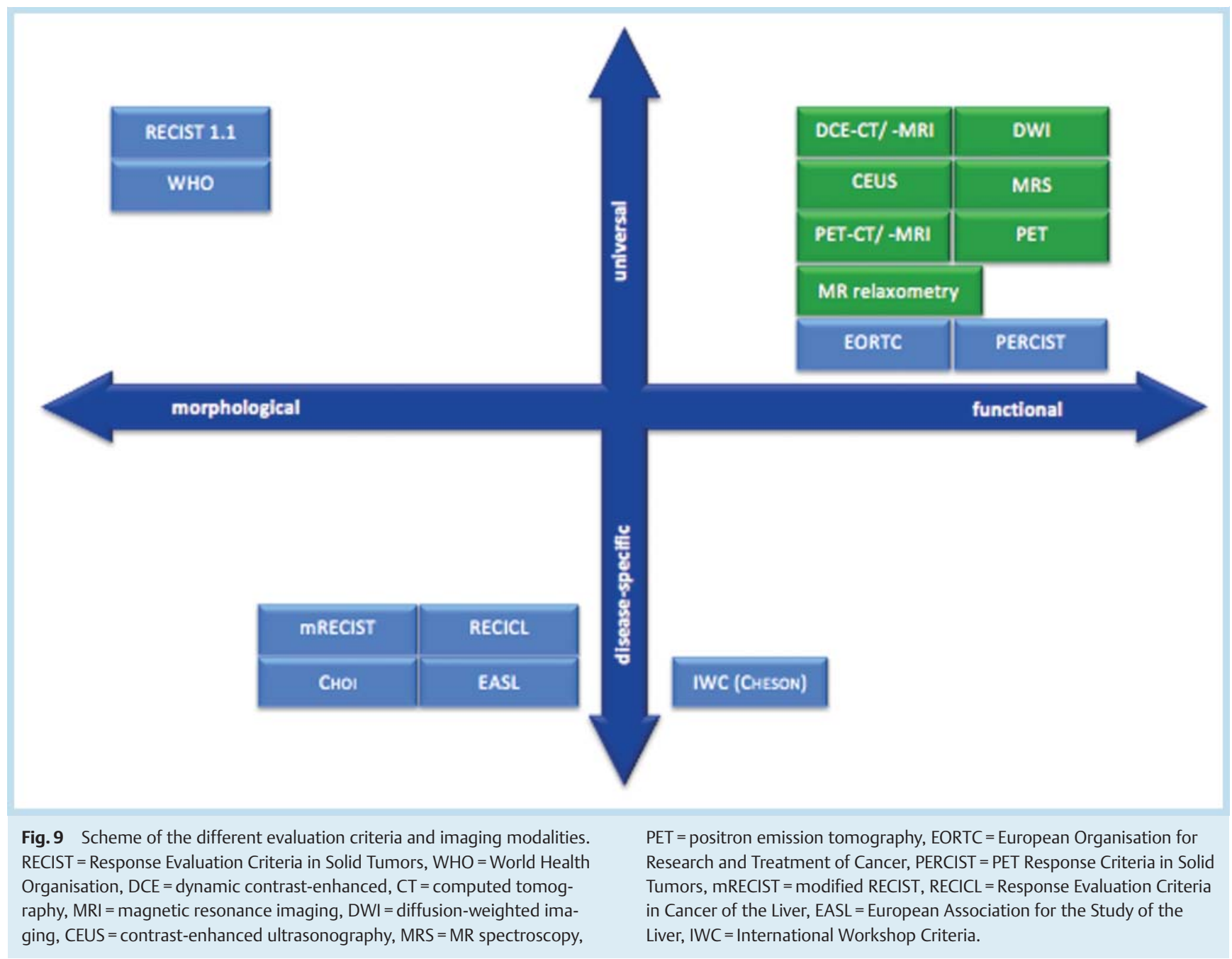




\section{References}

1 Gwyther SJ, Schwartz LH. How to assess anti-tumour efficacy by imaging techniques. Eur J Cancer 2008; 44: 39-35

2 Saini S. Radiologic measurement of tumor size in clinical trials: past, present and future. Am J Roentgenol 2001; 176: 333-334

3 Prasad SR, Jhaveri KS, Saini S et al. CT tumor measurement for therapeutic response assessment: comparison of unidimensional, bidimensional, and volumetric techniques initial observations. Radiology 2002; 225: 416-419

4 Stattaus J. Oncological imaging for therapy response assessment. Radiologe 2014; 54: 69-78

5 Rezai P, Pisaneschi MJ, Feng $C$ et al. A radiologist's guide to treatment response criteria in oncologic imaging: functional, molecular, and disease-specific imaging biomarkers. Am J Roentgenol 2013; 201: $246-256$

6 Weissleder R, Pittet MJ. Imaging in the era of molecular oncology. Nature 2008; 452: $580-589$

7 Eisenhauer EA, Therasse P, Bogaerts J et al. New response evaluation criteria in solid tumours: revised RECIST guideline (version 1.1). Eur J Cancer 2009; 45: 228 - 247

8 Chau CH, Rixe O, McLeod $\mathrm{H}$ et al. Validation of analytic methods for biomarkers used in drug development. Clin Cancer Res 2008; 14 : 5967-5976

9 World Health Organisation. WHO handbook for reporting results for cancer treatment. Geneva: 1979, http://whqlibdoc.who.int/offset/ WHO_OFFSET_48.pdf

10 Suzuki C, Jacobsson $H$, Hatschek T et al. Radiologic measurements of tumor response to treatment: practical approaches and limitations. Radiographics 2008; 28: 329-344

11 Buyse M, Thirion P, Carlson RW et al. Relation between tumour response to first-line chemotherapy and survival in advanced colorectal cancer: a meta-analysis. Meta-Analysis Group in Cancer. Lancet 2000; 356: $373-378$

12 Therasse P, Arbuck SG, Eisenhauer EA et al. New guidelines to evaluate the response to treatment in solid tumors. European Organization for Research and Treatment of Cancer, National Cancer Institute of the United States, National Cancer Institute of Canada. J Natl Cancer Inst 2000; 92: 205-216

13 Rezai $P$, Pisaneschi MJ, Feng $C$ et al. A radiologist's guide to treatment response criteria in oncologic imaging: anatomic imaging biomarkers. Am J Roentgenol 2013; 201: 237-245

14 Heckel F, Meine H, Moltz J et al. Segmentation-Based Partial Volume Correction for Volume Estimation of Solid Lesions in CT. IEEE Trans Med Imaging 2013, [Epub ahead of print]

15 Cademartiri F, Luccichenti G, Maffei E et al. Imaging for oncologic staging and follow-up: review of current methods and novel approaches. Acta Biomed 2008; 79: 85-91

16 Bornemann L, Kuhnigk JM, Dicken $V$ et al. Informatics in radiology (infoRAD): new tools for computer assistance in thoracic CT, part 2 therapy monitoring of pulmonary metastases. Radiographics 2005; 25: $841-848$

17 Buerke B, Puesken M, Müter S et al. Measurement accuracy and reproducibility of semiautomated metric and volumetric lymph node analysis in MDCT. Am J Roentgenol 2010; 195: 979-985

18 Weßling J, Puesken M, Koch R et al. MSCT follow-up in malignant lymphoma: comparison of manual linear measurements with semi-automated lymph node analysis for therapy response classification. Fortschr Röntgenstr 2012; 184: 795-804

19 Tirkes T, Hollar MA, Tann $M$ et al. Response criteria in oncologic imaging: review of traditional and new criteria. Radiographics 2013; 33: $1323-1341$

20 Layer G, Stahl T, Hoffend J. Bildgebende Beurteilung des Therapieansprechens unter Chemotherapie. Radiologie up2date 2013; 13: 221 239

21 Bruix J, Sherman M, Llovet JM et al. Clinical management of hepatocellular carcinoma. Conclusions of the Barcelona-2000 EASL conference. European Association for the Study of the Liver. J Hepatol 2001; 35: $421-430$

22 Lencioni R, Llovet JM. Modified RECIST (mRECIST) assessment for hepatocellular carcinoma. Semin Liver Dis 2010; 30: 52 -60

23 Kudo M, Kubo S, Takayasu K et al. Response Evaluation Criteria in Cancer of the Liver (RECICL) proposed by the Liver Cancer Study Group of Japan (2009 Revised Version). Hepatol Res 2010; 40: 686-692
24 Cheson BD, Horning SJ, Coiffier B et al. Report of an international workshop to standardize response criteria for non-Hodgkin's lymphomas: NCI sponsored International Working Group. J Clin Oncol 1999; 17: 1244

25 Cheson BD, Pfistner B, Juweid ME et al. Revised response criteria for malignant lymphoma. J Clin Oncol 2007; 25: 579-586

26 Choi H. Charnsangavej C, de Castro Faria S et al. CT evaluation of the response of gastrointestinal stromal tumors after imatinib mesylate treatment: a quantitative analysis correlated with FDG PET findings. Am J Roentgenol Am J Roentgenol 2004; 183: 1619-1628

27 Marcus CD, Ladam-Marcus V, Cucu C et al. Imaging techniques to evaluate the response to treatment in oncology: current standards and perspectives. Crit Rev Oncol Hematol 2008; 72: 217-238

28 Murukesh N, Dive C, Jayson GC. Biomarkers of angiogenesis and their role in the development of VEGF inhibitors. Br J Cancer 2010; 102: $8-18$

29 Zweifel M, Padhani AR. Perfusion MRI in the early clinical development of antivascular drugs: decorations or decision making tools? Eur J Nucl Med Mol Imaging 2010; 37: S164-S182

$30 \mathrm{Kim}$ KW, Lee JM, Jeon YS et al. Vascular disrupting effect of CKD-516: preclinical study using DCE-MRI. Invest New Drugs 2013; 31: 1097 1106

31 Persigehl T, Ring J, Bremer $C$ et al. Non-invasive monitoring of tumorvessel infarction by retargeted truncated tissue factor tTF-NGR using multi-modal imaging. Angiogenesis 2014; 17: 235-246

32 Miles KA, Griffiths MR. Perfusion CT: a worthwhile enhancement? $\mathrm{Br}$ J Radiol 2003; 76: 220-231

33 Wang $Q$, Shi $G$, Wang $L$ et al. Early prediction of response of Sorafenib on hepatocellular carcinoma by CT perfusion imaging: an animal study. Br J Radiol 2014; 87: 20130695

34 Goh V, Glynne-Jones R. Perfusion CT imaging of colorectal cancer. Br J Radiol 2014; 87: 20130811

35 Wilhelm T, Stieltjes B, Schlemmer HP. Whole-Body-MR-Diffusion Weighted Imaging in Oncology. Fortschr Röntgenstr 2013; 185: 950 958

36 Thoeny HC, Ross BD. Predicting and monitoring cancer treatment response with diffusion-weighted MRI. J Magn Reson Imaging 2010; 32: $2-16$

37 Padhani AR, Liu G, Koh DM et al. Diffusion-weighted magnetic resonance imaging as a cancer biomarker: consensus and recommendations. Neoplasia 2009; 11: $102-125$

38 Pokharel SS, Macura KJ, Kamel IR et al. Current MR imaging lipid detection techniques for diagnosis of lesions in the abdomen and pelvis. Radiographics 2013; 33: 681-702

39 Glunde K, Bhujwalla ZM, Ronen SM. Choline metabolism in malignant transformation. Nat Rev Cancer 2011; 11: 835-848

40 Persigehl T, Wall A, Kellert J et al. Tumor blood volume determination by using susceptibility-corrected DeltaR2* multiecho MR. Radiology 2010; 255: $781-789$

41 Contractor KB, Aboagye EO. Monitoring predominantly cytostatic treatment response with 18F-FDG PET. J Nucl Med 2009; 50: 97S$105 \mathrm{~S}$

42 Young H, Baum R, Cremerius $U$ et al. Measurement of clinical and subclinical tumour response using [18F]-fluorodeoxyglucose and positron emission tomography: review and 1999 EORTC recommendations. European Organization for Research and Treatment of Cancer (EORTC) PET Study Group. Eur J Cancer 1999; 35: 1773-1782

43 Wahl RL, Jacene H, Kasamon Y et al. From RECIST to PERCIST: Evolving Considerations for PET response criteria in solid tumors. J Nucl Med 2009; 50: $122 \mathrm{~S}-150 \mathrm{~S}$

44 Dinkel J, Khalilzadeh O, Hintze C et al. Inter-observer reproducibility of semi-automatic tumor diameter measurement and volumetric analysis in patients with lung cancer. Lung Cancer 2013; 82: 76-82

45 Weßling J, Schülke C, Koch R et al. Therapy Response Evaluation of Malignant Lymphoma in a Multicenter Study: Comparison of Manual and Semiautomatic Measurements in CT. Fortschr Röntgenstr 2014, [Epub ahead of print]

46 Gatenby RA, Grove O, Gillies RJ. Quantitative imaging in cancer evolution and ecology. Radiology 2013; 269: 8-15

47 Van Beers BE, Vilgrain V. Biomarkers in abdominal imaging. Abdom Imaging 2009; 34: 663-667 